\title{
Will adding acetaminophen (paracetamol) to ibuprofen be more effective in relieving postoperative pain on symptomatic necrotic teeth?
}

\author{
Abstracted from \\ Wells LK, Drum M, Nusstein J, Reader A, Beck M. \\ Efficacy of Ibuprofen and ibuprofen/acetaminophen on postoperative pain \section{treatment for pulpal necrosis is ibuprofen/ acetaminophen more effective in managing post-operative pain than ibuprofen alone?} \\ Question: In patients undergoing endodontic
} in symptomatic patients with a pulpal diagnosis of necrosis. J Endod 2011; 37: 1608-1612.

Address for correspondence: Dr Melissa Drum, Division of Endodontics, College of Dentistry,

The Ohio State University, 305 West 12th Avenue, Columbus, OH 43210, USA. E-mail: drum.13@osu.edu treatment allocation.

\section{Design Randomised controlled trial}

Intervention Patients with a clinical diagnosis of a symptomatic tooth with a pulpal diagnosis of necrosis and moderate to severe pain at the time of treatment were randomised to either 80 capsules of $150 \mathrm{mg}$ ibuprofen or 80 capsules of $150 \mathrm{mg}$ ibuprofen/250mg acetaminophen. Patients were to take four capsules every six hours. Escape medication (hydrocodone/acetaminophen) was also available; if patients had significant swelling or fever, they were seen clinically. Patients' preoperative pain was assessed on the/a visual analogue scale (VAS). Patients also received a six-day diary to be completed after anaesthesia wore off and every morning for five days, to record pain, symptoms and the number of capsules taken.

Outcome measure The main outcome measures were pain and medication use.

Results No significant differences were found between the two groups. Conclusions There were decreases in pain levels and analgesic use over time for the ibuprofen and ibuprofen/acetaminophen groups. There was no statistically significant difference between the two groups for analgesic use or escape medication use. Approximately $20 \%$ of patients in both groups required escape medication to control pain.

\section{Commentary}

This randomised control study compares the effectiveness of $600 \mathrm{mg}$ of ibuprofen against the same dose of ibuprofen plus 1000mg of acetaminophen on the management of postoperative pain of symptomatic teeth diagnosed with pulpal necrosis in otherwise healthy adults over five days.

Seventy-one patients with moderate to severe pain measured according to the Heft-Parker VAS scale and confirmed diagnosis of necrosis with at least a $2 \mathrm{~mm}$ radiolucency present were enrolled to allow for detecting a $30 \%$ difference among groups based on the power calculation. Randomisation was used to generate the numeric coding displayed on the medication bottles. Patients and the researcher providing the medication were blinded to the

The two groups were similar in all characteristics but the initial pain was higher in the combination group.

Patients were instructed to take the medication every four hours and kept a five-day diary to record any pain experience. No difference was observed in the decrease of pain rating and the number of pills used between groups. Pain elimination was not achieved in any group even after five days.

Rescue medication was available after contacting the researcher. Hydrocodone/acetaminophen $5 \mathrm{mg} / 500 \mathrm{mg}$ was given with the indication to discontinue the study medication. Rescue medication usage did not show a statistically significant difference between the groups: $20 \%$ of the patients in the ibuprofen group and in 19\% of the combination group required it.

Antibiotics were prescribed based on the presence of significant swelling or fever. All patients requiring antibiotics also used rescue medication (six in the ibuprofen and one in the combination group)

The study did not include a true placebo group and side effects were not assessed. The number of patients needing rescue medication may under-power the ability to detect any difference. The use of a fixed pain medication combination is an attractive option that capitalises on potential synergism and decreases side effects.

In this study no differences were found between the two regimens, still, if there was a significant benefit in the combination, a daily amount of 4 grams of acetaminophen is no longer recommended even in healthy individuals because of the potential for liver damage (http://www.fda.gov/downloads/ Drugs/GuidanceComplianceRegulatoryInformation/Guidances/ UCM310477.pdf ).

\section{Practice point}

- Adding acetaminophen to a regimen of $600 \mathrm{mg}$ of ibuprofen every six hours does not seem to provide any additional benefit for pain relief to symptomatic necrotic teeth.

Silvia Spivakovsky

NYU College of Dentistry, New York, USA

Evidence-Based Dentistry (2012) 13, 105. doi:10.1038/sj.ebd.6400889 